\title{
Severe perinatal hypophosphatasia case with a novel mutation
}

\author{
Havva Yazicia (D), Ebru Canda ${ }^{a}$ (D), Sema Kalkan Ucara (D), Mahmut Cokera
}

\begin{abstract}
Hypophosphatasia (HPP) is a rare inherited disorder caused by mutations in the ALPL gene. Mineralization defect in bones and teeth, abnormal respiratory function, seizures, hypotonia, bone pain, and nephrocalcinosis can be observed. Clinical forms are usually recognized based on age at diagnosis and severity of features.

We present an infant with an enlarged anterior fontanelle, soft calvarium, fractures, respiratory distress, and seizures. Biochemical analysis showed hypercalcemia, normal serum phosphate, and low serum alkaline phosphatase (ALP) levels. X-ray showed hypomineralization, fractures, and callus formations. Plasma pyridoxal 5'-phosphate (PLP) was $762 \mathrm{mg} / \mathrm{L}$ (NV : 5-50) and urine phosphoethanolamine (PEA) was $1015 \mathrm{mmol} / \mathrm{L}(\mathrm{NV}: 15-341)$ and ALPL gene analysis showed two compound heterozygous mutations, one of which is a novel one. Early diagnosis and treatment of perinatal HPP may improve outcomes and might have a positive impacton survival. Key words: hypophosphatasia, ALPL gene, asfotase alfa, enzyme replacement therapy.
\end{abstract}

http:/ / dx.doi.org/10.5546/ aap.2022.eng.e21

To cite: Yazici H, Canda E, Kalkan Ucar S, Coker M. Severe perinata hypophosphatasia case with a novel mutation. Arch Argent Pediatr 2022;120(1):e21-e24.

\section{INTRODUCTION}

Hypophosphatasia (HPP) is a rare disease of the bone and mineral metabolism due to the low activity of the tissue nonspecific isoenzyme of alkaline phosphatase (TNSALP) caused by mutations in the gene encoding TNSALP.

a. Department of Pediatric Metabolism and Nutrition, Ege University Faculty of Medicine, Izmir, Turkey.

E-mail address:

Havva Yazici: havvaya@gmail.com

Funding: None.

Conflict of interest: None.

Received: $12-9-2020$

Accepted: 7-13-2021
In 1923, ALP was first defined, and the first HPP case report was in $1948 .{ }^{1}$ HPP reflects the defective activity of TNSALP. Low serum ALP activity is the primary and easily detectable first biochemical feature of HPP. There are only a few studies that report HPP incidence and prevalence. Early-onset forms (perinatal, infantile) of HPP occur in approximately 1 per 100,000 and 300,000 births in Canada and Europe, respectively. ${ }^{2}$ The clinical spectrum is notably broad: It extends from neonatal death to dental problems manifesting with or without bone disease in adult life. Clinical forms, which are distinguished by the age of onset at HPP presentation, have been described: "adult," "childhood," "infantile," and "perinatal" HPP. The clinical manifestations of perinatal HPP in utero, are similar to other skeletal dysplasias. Caput membranaceum, shortened limbs, chest deformity and stillbirth are clinical features at birth. Apnea, respiratory insufficiency, vitamin B6-dependent seizures, and fractures are other clinical findings in the neonatal period..$^{1-3}$

Until very recently, severe perinatal HPP was lethal at birth or in the first months of life. Subcutaneous asfotase alfa (STRENSIQ ${ }^{\circledR}$, Alexion Pharmaceuticals, Inc., Boston, MA, USA) is a bone-targeted human recombinant enzyme replacement therapy. It is approved for treatment in HPP patients and had improved bone mineralization, survival, and ventilation-free survival. ${ }^{1,4,5}$ Perinatal HPP should be considered in the differential diagnosis of patients with skeletal deformities, convulsions, hypercalcemia, and low ALP. Early diagnosis and treatment of perinatal HPP may improve outcomes and might have a positive impact on survival.

This is a clinical report of a case diagnosed with HPP in the newborn period.

\section{CASE}

A girl was born to healthy nonconsanguineous parents at 38-week gestational age after cesarean delivery. Her birth weight was $2800 \mathrm{~g}$. She had a large anterior fontanelle, soft calvarium, narrow bell-shaped chest, and bowing in both forearms on her first examination. She presented tachypnea, subcostal retractions, 
and grunting. Nasal continuous positive airway pressure was required for five days. There was no need for oxygen support afterward. On day six, she had a seizure. Serial electroencephalogram tracing was performed. Burst suppression pattern and multifocal epileptic activity were detected. She was treated with intravenous phenytoin at a $20 \mathrm{mg} / \mathrm{kg}$ loading dose and a $5 \mathrm{mg} / \mathrm{kg}$ maintenance dose. She had no convulsions on the following days.

Her complete blood count was normal. Low serum ALP level (10 U/L, N: > 70) was notable on biochemical investigations. Reference ranges for ALP by age are in Table $1 .^{6}$ Serum calcium was high (13.5, mg/dl; N : 9-10.9), and serum phosphate was normal (4.9 mg/ dl; $\mathrm{N}: 3.4-5.9)$. Serum 25-hydroxy vitamin D3 was normal $(96 \mathrm{nmol} / \mathrm{L}$, the cut-off for vitamin D3 deficiency is $<25$ ) and parathyroid hormone was low ( $3 \mathrm{pg} /$ $\mathrm{ml} ; \mathrm{N}$ : 11-67). Spot urine calcium/creatinine ratio was normal $(0,6 \mathrm{mg} / \mathrm{mg}$; $\mathrm{N}:<0,8)$.

Skeletal radiographs revealed radiolucent bone structures and thin ribs. Fractures of radius and ulna, and callus formations were also detected. Cranial ultrasonography was normal. Bilateral nephrocalcinosis was seen on abdominal ultrasound at the age of 14 days. Cranial tomography was normal.

Plasma pyridoxal 5'-phosphate (PLP) and urine phosphoethanolamine (PEA) levels were high $762 \mathrm{ug} / \mathrm{L}(\mathrm{N}: 5-50)$ and $1015 \mathrm{umol} / \mathrm{L}$ (N : 15341), respectively. The suspected diagnosis was severe perinatal HPP and was confirmed by biochemical determination of PLP and PEA. ALPL gene analysis showed a compound heterozygosis for p.R184W (c.550C > T)/p.G288A (c.863G>C).

TABLE 1. Reference ranges for alcaline phosphatase levels $(U / L)$ by age $e^{6}$

\begin{tabular}{lcc}
\hline Age & Male & Female \\
\hline$<1$ month & 60 & 60 \\
1-11 months & 70 & 70 \\
1-3 years & 125 & 125 \\
4-11 years & 150 & 150 \\
12-13 years & 160 & 110 \\
14-15 years & 60 & 40 \\
$\geq 20$ years & 40 & 40 \\
\hline
\end{tabular}

Patient was treated with subcutaneous $2 \mathrm{mg} /$ $\mathrm{kg} /$ dose asfotase alfa for the first time on the 60th day of life. The treatment continued three times a week. The first two doses of asfotase alfa were administered at the hospital, with no injection site reaction. The other adverse events, hypocalcemia, hyperphosphatemia, and calcification, were not observed in our patient, either. Nasogastric feeding was recommended to the family due to her hypotonicity, but the family refused. She was discharged from the hospital when she was 64 days old. After the 5th dose of subcutaneous asfotase alfa, when she was 72 days old, her parents found her lying in bed unresponsive and not breathing. The family went to the nearby health center, and the health institute declared that the patient had died. The autopsy could not be examined because the family did not give the necessary legal permission for the autopsy. Therefore, we could not obtain data in terms of aspiration, which may cause death in hypotonic infants.

\section{DISCUSSION}

Perinatal HPP is the most severe form of the disease. Fractures and calluses were detected in our patient in the first days of her life, indicating that the condition started in the intrauterine period. Respiratory distress was also seen in the first days of life. Perinatal HPP is associated with significant mortality and must be distinguished from "benign prenatal," which refers to skeletal deformity in utero or birth ex utero that improves ex utero spontaneously. ${ }^{1}$

Our patient required respiratory support for five days. Mortality is as high as 50\%-100 \% in perinatal HPP, consequence of respiratory failure due to hypoplastic lungs and functional defects secondary to deformity of the chest. . $^{1,45,7}$

In the presented case, skeletal deformities, low ALP, hypercalcemia, normal serum 25-hydroxy vitamin D3 and low parathyroid hormone strongly suggested HPP. Elevated plasma PLP and elevated urine PEA are the biochemical hallmarks in this case. Although not measured in this patient, inorganic pyrophosphate $(\mathrm{PPi})$ is one of the most significant mineralization inhibitors. ${ }^{6}$

Differential diagnosis includes rickets, neglect, non-accidental trauma, osteogenesis imperfecta type II, thanatophoric dysplasia, campomelic dysplasia, and chondrodysplasias with bone mineralization defects. ${ }^{1,3,6}$ 
Pyridoxine-dependent seizures are usually a fatal prognostic indicator. PLP is one of the significant intracellular bioactive forms of vitamin B6. If PLP cannot be dephosphorylated to pyridoxal (PL) to cross the blood-brain barrier, then vitamin B6-dependent seizures may occur. ${ }^{1,3,5}$ We could control the convulsion of our patient without the need for pyridoxine.

Nephrolithiasis can be observed due to hypercalcemia and / or hypercalciuria. ${ }^{1,3,4}$ Bilateral nephrocalcinosis was revealed on abdominal ultrasound. Our patient had hypercalcemia.

ALPL gene analysis revealed a compound heterozygous mutation: p.R184W (c.550C >T)/ p.G288A (c.863G>C). The p.R184W (c.550C >T) mutation is experimentally associated with $0.6 \%$ of wild-type enzyme activity, prognosticating a severe phenotype. ${ }^{1,7-9}$ The p.G288A (c.863G>C) mutation has not been previously reported to the best of our knowledge. This expressivity is largely explained by autosomal recessive (AR) vs. autosomal dominant (AD) transmission of at least 275 mutations within $A L P L$, with $74 \%$ being missense. AR inheritance usually explains severe HPP, whereas both AD and AR inheritance is related to mild HPP. ${ }^{3}$ Parental testing was confirmed for two variants by targeted sequencing of ALPL. Her mother's and father's ALP levels were normal, $86 \mathrm{U} / \mathrm{L}$ and $73 \mathrm{U} / \mathrm{L}$, respectively.

The first pharmacological therapeutic option is the bone-targeted enzyme-replacement therapy; asfotase alfa has been indicated for long-term enzyme replacement therapy in patients with pediatric-onset hypophosphatasia to treat the bone manifestations of the disease. ${ }^{1,5,7}$ Enzyme replacement therapy can improve survival. In 2016, Whyte et al., reported improved survival in treated perinatal and infantile HPP patients vs. historical controls: $95 \%$ vs. $42 \%$ at age one year and $84 \%$ vs. $27 \%$ at age five years, respectively. ${ }^{4}$ Clinical trials also reported the safety and improvement of mineralization and respiratory function. . $, 5,7^{-1}$

The most frequent adverse event on asfotase alfa treatment is an injection site reaction. In our patient, there were no local reactions. The other adverse events, such as hypocalcemia, hyperphosphatemia, and ectopic calcification, were not observed in our patient, either. ${ }^{4,5,7}$
Congenital hypophosphatasia should occur in the differential diagnosis in the presence of multiple system findings such as skeleton deformity, spontaneous fractures, hypotony, respiratory distress, and seizure. The positive effects on bone mineralization have been reported at four weeks after starting treatment, and it may take months to see improvement in respiratory function..$^{4,5}$ In our patient, the duration of treatment was insufficient to assess lung and bone response.

Our case again emphasizes the importance of beginning treatment as soon after birth as possible. Finally, earlier diagnosis of perinatal HPP with prenatal recognition and starting ERT as quickly as possible after delivery may improve outcomes and might positively impact survival. In addition, p.G288A change determined in our case is a newly identified alteration, to the best of our knowledge.

\section{Disclosure:}

The authors have indicated they have no financial relationships relevant to this article to disclose.

Alexion Pharmaceuticals Inc. provided a scientific/medical review of the case report as a courtesy upon authors' request; authors had final authority over all content in this publication.

\section{REFERENCES}

1. Whyte MP.Hypophosphatasia: an overview for 2017. Bone. 2017; 102:15-25.

2. Vogt M, Girschick H, Schweitzer T, Benoit C, et al. Pediatric hypophosphatasia: lessons learned from a retrospective single-center chart review of 50 children. Orphanet J Rare Dis. 2020; 15(1):212.

3. Whyte MP, Zhang F, Wenkert D, McAlister WH, et al. Hypophosphatasia: validation and expansion of the clinical nosology for children from 25 years experience with 173 pediatric patients. Bone. 2015; 75:229-39.

4. Whyte MP, Rockman-Greenberg C, Ozono K, Riese R, et al. Asfotase alfa treatment improves survival for perinatal and infantile hypophosphatasia. J Clin Endocrinol Metab. 2016; 101(1):334-42.

5. Kitaoka T, Tajima T, Nagasaki K, Kikuchi T, et al. Safety and efficacy of treatment with asfotase alfa in patients with hypophosphatasia: results from a Japanese clinical trial. Clin Endocrinol (Oxf). 2017; 87(1):10-9.

6. Simmons J. Best practices in: recognizing and diagnosing hypophosphatasia. Clinical Endocrinology News. 2013. [Accessed on: july 6, 2021]. Available in: https:// www.mdedge.com/endocrinology/article/78038/bestpractices-recognizing-and-diagnosing-hypophosphatasia

7. Scott LJ. Asfotase alfa in perinatal/infantile-onset and juvenile-onset hypophosphatasia: a guide to its use in the USA. BioDrugs. 2016; 30(1):41-8. 
8. Mornet E, Taillandier A, Peyramaure S, Kaper F, et al. Identification of fifteen novel mutations in the tissuenonspecific alkaline phosphatase (TNSALP) gene in
European patients with severe hypophosphatasia. Eur J Hum Genet. 1998; 6(4):308-14.

9. Sağlam H, Erdöl Ş, Dorum S. Clinical and genetic findings of Turkish hypophosphatasia cases. J Clin Res Pediatr Endocrinol. 2017; 9(3):229-36. 